\title{
System Of Record Name
}

National Cancer Institute

\section{Source}

National Cancer Institute. System Of Record Name. NCI Thesaurus. Code C94168.

A non-unique textual identifier for the system that assigned the identifier. 